\title{
Iptakalim Alleviates Rotenone-Induced Degeneration of Dopaminergic Neurons through Inhibiting Microglia-Mediated Neuroinflammation
}

\author{
Fang Zhou', , Jia-Yong Wu ${ }^{1,2}$, Xiu-Lan Sun', Hong-Hong Yao', Jian-Hua Ding' and Gang Hu*,' \\ 'Laboratory of Neuropharmacology, Department of Anatomy, Histology and Pharmacology, Nanjing Medical University, Nanjing, Jiangsu, \\ PR China
}

\begin{abstract}
Inhibition of microglia-mediated neuroinflammation has been regarded as a prospective strategy for treating neurodegenerative disorders, such as Parkinson's disease (PD). In the present study, we demonstrated that systematic administration with iptakalim (IPT), an adenosine triphosphate (ATP)-sensitive potassium channel $\left(K_{\text {ATP }}\right)$ opener, could alleviate rotenone-induced degeneration of dopaminergic neurons in rat substantia nigra along with the downregulation of microglial activation and mRNA levels of tumor necrosis factor- $\alpha$ (TNF- $\alpha$ ) and cyclooxygenase-2 (COX-2). In rat primary cultured microglia, pretreatment with IPT suppressed rotenone-induced microglial activation evidenced by inhibition of microglial amoeboid morphological alteration, declined expression of EDI (a marker for activated microglia), and decreased production of TNF- $\alpha$ and prostaglandin E2 (PGE 2 ). These inhibitory effects of IPT could be reversed by selective mitochondrial $K_{\text {ATP }}$ (mito $K_{\text {ATP }}$ ) channel blocker 5-hydroxydecanoate (5-HD). Furthermore, pretreatment with IPT prevented rotenone-induced mitochondrial membrane potential loss and p38/c-jun N-terminal kinase (JNK) mitogen-activated protein kinase (MAPK) activation in microglia, which might in turn regulate microglial activation and subsequent production of TNF- $\alpha$ and $\mathrm{PGE}_{2}$. These data strongly suggest that the $K_{\mathrm{ATP}}$ opener IPT may be a novel and promising neuroprotective drug via inhibiting microgliamediated neuroinflammation.

Neuropsychopharmacology (2007) 32, 2570-2580; doi:I0.I038/sj.npp. I30 I38I; published online I4 March 2007
\end{abstract}

Keywords: iptakalim; ATP-sensitive potassium channel; microglia; rotenone; neuroinflammation; Parkinson's disease

\section{INTRODUCTION}

Microglia, the resident immune cells in the brain, play a pivotal role in immune surveillance of central nervous system (CNS). Increasing evidence has demonstrated that microglia-mediated neuroinflammatory process is critically involved in the initiation and development of neurodegenerative disorders, such as Parkinson's disease (PD; Block and Hong, 2005; Minghetti, 2005). When subjected to abnormal stimulation, such as neurotoxins, neuronal debris, or injury, microglia become gradually activated and produce a host of factors, including tumor necrosis factor- $\alpha$ (TNF- $\alpha)$, prostaglandin E2 $\left(\mathrm{PGE}_{2}\right)$, interleukin- $1 \beta$ (IL-1 $\beta$ ), nitric oxide (NO), and reactive oxygen species (ROS). Accumulation of these proinflammatory and

* Correspondence: Professor G Hu, Laboratory of Neuropharmacology, Department of Anatomy, Histology and Pharmacology, Nanjing Medical University, 140 Hanzhong Road, Nanjing, Jiangsu 210029, PR China, Tel: + 8602586863 I69, Fax: + 8602586863108 ,

E-mail: ghu@njmu.edu.cn

${ }^{2}$ These authors contributed equally to this work.

Received I August 2006; revised 12 January 2007; accepted 20 January 2007 cytotoxic factors is deleterious directly to neurons and subsequently induces further activation of microglia, resulting in a vicious cycle (Block and Hong, 2005; Herrera et al, 2005). Thus, inhibition of microglial activation and subsequent inflammatory process may provide therapeutic benefits for inflammation-related neurodegenerative disorders, such as PD (Gao et al, 2003b). Moreover, the compounds possessing anti-neuroinflammatory properties have been becoming one of the most promising candidates for PD therapy.

ATP-sensitive potassium channels ( $K_{\mathrm{ATP}}$ channels), which belong to a class of inwardly rectifying potassium channels, are activated by a decrease in ATP/ADP ratio. Activation of $K_{\mathrm{ATP}}$ channels induces cell hyperpolarization, which may reduce cell activity and energy consumption, thereby linking metabolic state to excitability (Rodrigo and Standen, 2005). $K_{\text {ATP }}$ channels are composed of discrete poreforming (Kir6.1/Kir6.2) and regulatory subunits (SUR1/ SUR2). These channels in the brain do not belong to a homogenous group, and different combinations of possible $K_{\mathrm{ATP}}$ channel subunits give rise to functional $K_{\mathrm{ATP}}$ channels with a variety of biophysical, pharmacological and metabolic properties (Liss and Roeper, 2001). It is well 
documented that $K_{\mathrm{ATP}}$ channel openers, especially mitochondrial $K_{\mathrm{ATP}}$ channel (mito $K_{\mathrm{ATP}}$ channel) openers, can provide neuroprotective effects for neuron and astrocyte against ischemia, trauma, and neurotoxicants (Busija et al, 2004; Hu et al, 2005; Yamada and Inagaki, 2005; Yang et al, 2006). The result from our laboratory first reported BV-2 microglial cell line expressed Kir6.1/SUR2 $K_{\text {ATP }}$ channels, which could regulate BV-2 cell activation and the production of inflammatory factors (Liu et al, 2006). Recently, we found that opening $K_{\mathrm{ATP}}$ channels expressed in rat primary cultured microglia prevented dopaminergic neurons from rotenone-induced neurodegeneration via inhibiting microglial activation (not published). As most developed $K_{\text {ATP }}$ channel openers fail to pass through the blood-brain barrier, there are few effective $K_{\text {ATP }}$ channel openers to be used in vivo for investigating the role of $K_{\mathrm{ATP}}$ channels in CNS in neurodegenerative disorders.

Iptakalim (IPT) is a lipophilic para-amino compound, which has been demonstrated to be a novel $K_{\mathrm{ATP}}$ channel opener by pharmacological, electrophysiological and biochemical studies, and receptor binding test (Yang et al, 2004, 2005; Xie et al, 2005; Wang et al, 2006). Our previous studies have revealed that IPT exhibited significant neuroprotective effects not only on behavioral symptoms, but also on neuronal necrosis and apoptosis in different animal models of stroke, PD, as well as in cultured cells. And these effects of IPT were supposed mainly through activation of mito $K_{\mathrm{ATP}}$ channels (Wang et al, 2004, 2005, 2006; Yang et al, 2004, 2005, 2006; Hu et al, 2005; Liu et al, 2006). Most recently, we found that IPT exhibited potent neuroprotective effects against rotenone-induced neurotoxicity by inhibiting inducible nitric oxide synthase (iNOS) expression and activity (Yang et al, 2005, 2006). Notably, as iptakalim can freely get through the bloodbrain barrier and has few effects on peripheral blood pressure, it may be a prospective agent for treating PD and other neurodegenerative diseases. However, it needs to be further investigated whether IPT exerts neuroprotection via inhibiting microglial activation and the sequential neuroinflammation.

Rotenone, a classic and high-affinity inhibitor of mitochondrial complex I, can induce selective dopamine neurons degeneration in substantia nigra (Helmuth, 2000; Jenner, 2001; Alam and Schmidt, 2002), and $K_{\mathrm{ATP}}$ channel is considered as a potential downstream target of mitochondrial complex I inhibition (Liss et al, 2005). It is notable that microglial activation and subsequent inflammatory process induced by rotenone have been suggested to be the predominant mechanisms for degeneration of dopaminergic neurons in vivo and in vitro (Gao et al, 2002, 2003a, b; Perier et al, 2003; Sherer et al, 2003). Therefore, in the present study, we tried to explore whether the $K_{\mathrm{ATP}}$ channel opener IPT could inhibit microglia-mediated neuroinflammation induced by rotenone and thus prevent dopaminergic neurodegeneration.

\section{MATERIALS AND METHODS}

\section{Animals and Treatments}

Male Sprague-Dawley rats aged 7 weeks (220-240 g) were chosen for experiments. Five rats were kept in a cage under standard laboratory conditions with free access to standard laboratory food and tap water, constant room temperature of $22^{\circ} \mathrm{C}, 50-60 \%$ humidity, and a natural day-night cycle. All experiments were performed according to the National Institutes of Health Guide for the Care and Use of Laboratory Animals (publication no. 85-23, revised 1985) and the Guidelines for the Care and Use of Animals in Neuroscience Research by the Society for Neuroscience and approved by IACUC (Institutional Animal Care and Use Committee of Nanjing Medical University).

Dimethylsulfoxide (DMSO)/polyethylene glycol (1:1) was used as vehicle for rotenone. IPT was dissolved in sterile saline. Diazoxide was dissolved in $100 \%$ DMSO as vehicle and diluted with sterile saline to a concentration of $1 \mathrm{mg} / \mathrm{ml}$ $(0.2 \%$ DMSO) for administration to rats. Rats were randomly divided into five groups. Control rats $(n=16)$ received vehicle only. Rats of model group $(n=20)$ were subcutaneously injected with rotenone $(2.5 \mathrm{mg} / \mathrm{kg} / \mathrm{day})$ for 4 weeks and daily dose of rotenone was administered t.i.d. at 08:00, 14:00, and 20:00, respectively. The injection volume was $0.1 \mathrm{ml} / 100 \mathrm{~g}$ body weight. Other rats were received intragastric administration with IPT $(1.5,3.0 \mathrm{mg} / \mathrm{kg} / \mathrm{day}$, $n=16)$ or diazoxide $(3.0 \mathrm{mg} / \mathrm{kg} /$ day, $n=16)$ for 3 days. On day 4, IPT or diazoxide was pretreated 1 hour before the injection with rotenone $(2.5 \mathrm{mg} / \mathrm{kg} / \mathrm{day}$, subcutaneously) on a daily basis for 4 weeks.

Rats of rotenone group underwent an obvious mortality (40\%) after 4 week treatments. Twelve to 16 animals remained per group. After the last behavior test, rats were killed and their brains were used for morphological $(n=6)$ and biochemical $(n=4)$ analyses.

\section{Behavioral Tests}

The catalepsy test and rotarod test were performed to evaluate the effects of IPT and diazoxide on rotenoneinduced parkinsonian symptoms. All rats were tested for catalepsy and rotarod at five time points, before administration and 1, 2, 3, and 4 weeks after treatments. Only rats that completed all 4 weeks of the experiments were included in statistical analyses $(n=12)$. For catalepsy test, rats were placed with both forepaws on bars $9 \mathrm{~cm}$ above and parallel from the base and were in a half-rearing position. Latency time of the removal of the paw was recorded. The basic requirement is for rotating roller (or rotarod), consisting of (1) a roller of the appropriate diameter $(8 \mathrm{~cm})$ for rats, (2) a power source for turning the roller (control of rod speed), and (3) four circular separators placed along the rod at suitable intervals to divide the roller into equal-sized compartments for simultaneous testing of four animals. For the rotarod test, rats were placed on the rod and sequentially tested at 5, 10, and 15 r.p.m. for a maximum of $300 \mathrm{~s}$ each speed. The length of time that each animal was able to stay on the rod at each rotarod speed was recorded.

\section{Microglia-Enriched Cultures}

Rat primary microglial cells were prepared according to previously described protocol with slight modifications (Gao et al, 2002). Briefly, tissues from whole brains of postnatal (P1-P2) Sprague-Dawley rats were triturated and 
then cells were plated on poly-D-lysine precoated cell culture flasks in DMEM containing $10 \%$ fetal calf serum, $100 \mathrm{U} / \mathrm{ml}$ penicillin, and $100 \mu \mathrm{g} / \mathrm{ml}$ streptomycin. Cultures were maintained at $37^{\circ} \mathrm{C}$ in a humidified atmosphere of $5 \%$ $\mathrm{CO}_{2} / 95 \%$ air. After reaching a confluent monolayer of glial cells (10-14 days), microglia were separated from astrocytes by shaking off for $5 \mathrm{~h}$ at 100 r.p.m. and replated on 24-well culture plates at a density of $10^{5} \mathrm{cells} / \mathrm{cm}^{2}$. After plating the microglia-enriched population for $24 \mathrm{~h}$, cells were treated with rotenone, $K_{\text {ATP }}$ channel openers, blocker, or vehicle alone. The enriched microglia were $>98 \%$ pure as determined by OX-42-IR (a marker for microglia; Serotec, USA) and GFAP-IR (a marker for astrocyte; Sigma, USA).

\section{Immunochemistry and Stereology}

Animals were perfused with $4 \%$ paraformaldehyde, and brains were dissected out and maintained in $4 \%$ paraformaldehyde overnight. Brains were cryopreserved in 30\% sucrose in phosphate buffered saline (PBS) and then stored at $-70^{\circ} \mathrm{C}$ until used. Free-floating sections encompassing the entire midbrain were prepared using a cryostat. For TH, OX-42, and ED1 immunostaining, tissue sections $(30 \mu \mathrm{m})$ were incubated with primary antibodies overnight at $4{ }^{\circ} \mathrm{C}$. Primary antibodies used in this study were as follows: mouse anti-TH (1:4000; Sigma, USA), mouse anti-OX-42 (1:100; Serotec, USA), mouse anti-ED1 (1:100; Serotec, USA). Immunostaining was visualized by using $3,3^{\prime}-$ diaminobenzidine. Sections were then counterstained with hematoxylin.

The total number of TH-and hematoxylin-eosin-positive neurons in substantia nigra were counted from six rats per group by using the optical fractionator method (West, 1993), an unbiased method of cell counting that is not affected by either the volume of reference (ie $\mathrm{SNpc}$ ) or the size of the counted elements (ie neurons) (Stereoinvestigator, MicroBrightField). In agreement with this method, TH- and hematoxylin-eosin-stained neurons were counted in the right and left SNpc of every fourth section $(30 \mu \mathrm{m})$ throughout the entire extent of the SNpc. Each midbrain section was viewed at low power ( $\times 10$ objective), and the $\mathrm{SNpc}$ was outlined according to the established anatomical landmarkers (the rat brain atlas by Paxinos and Watson, 1997). Then at a random start, the number of TH- and hematoxylin-eosin-stained cells was counted at high power ( $\times 100$ oil). To avoid double counting of neurons with unusual shapes, TH- and hematoxylin-eosin-stained cells were counted only when their nuclei were optimally visualized, which occurred only in one focal plane. After all of the TH- and hematoxylin-eosin-positive neurons were counted, the total numbers of TH- and hematoxylin-eosinpositive neurons in the SNpc were calculated by using the formula described by West (1993). Sampling grid dimensions were $120 \times 120 \times 5 \mu \mathrm{m}$ ( $x, y$, and $z$ axes).

Cells were fixed using $4 \%$ paraformaldehyde followed by blocking with PBS containing $10 \%$ bovine serum albumin (BSA). After blocking, cells were incubated at $4{ }^{\circ} \mathrm{C}$ overnight with the primary antibody ED1 (1:100, a marker for activated microglia). Stained cells were observed by scanning confocal microscopy, and the total ED1 fluorescence intensities of every well were quantitated using imageanalysis software (Simple PCI).

\section{Reverse Transcription-Polymerase Chain Reaction}

Total RNA was extracted from selected brain regions after drug treatment using Trizol (Invitrogen Life Technologies, USA). $2 \mu \mathrm{g}$ Two micrograms of total RNA was reversetranscribed into single-stranded cDNA. Polymerase chain reaction (PCR) was performed on the equivalent cDNAs from each sample. Amplification was performed with the following primer sets: (1) TNF- $\alpha$ (GenBank accession no. X66539): $5^{\prime}$-CAGACCCTCACACTCAGATCATCTT-3' (forward) and $5^{\prime}$-CAGAGCAATGACTCCAAAGTAGACCT-3' (reverse); (2) cyclooxygenase-2 (COX-2) (GenBank accession no. S67722): $5^{\prime}$-TGATGACTGCCCAACTCCCATG-3' (forward) and 5'-AAT GTTGAAGGTGTCCGGCAGC-3' (reverse); and (3) glyceraldehyde 3-phosphate dehydrogenase (GAPDH) (GenBank accession no. BC059110) 5'-TGGTGCCAAAAGGGTCATCT CC-3' (forward) and 5'-GCCAGCCCCAGCATCAAAGGTG-3' (reverse). The thermal cycling conditions of PCR were as follows: TNF- $\alpha$ and COX-2: $95^{\circ} \mathrm{C}, 5 \mathrm{~min} ; 95^{\circ} \mathrm{C}, 30 \mathrm{~s} ; 63^{\circ} \mathrm{C}, 30 \mathrm{~s}$; $72^{\circ} \mathrm{C}, 30 \mathrm{~s} ; 72^{\circ} \mathrm{C}, 10 \mathrm{~min}$ ( 35 cycles); GAPDH: $95^{\circ} \mathrm{C}, 5 \mathrm{~min}$; $95^{\circ} \mathrm{C}, 30 \mathrm{~s} ; 60^{\circ} \mathrm{C}, 30 \mathrm{~s} ; 72^{\circ} \mathrm{C}, 30 \mathrm{~s} ; 72^{\circ} \mathrm{C}, 10 \mathrm{~min}$ (30 cycles). The PCRs were then visualized on a $1.8 \%$ agarose gel containing $0.06 \mu \mathrm{g} / \mathrm{ml}$ ethidium bromide and analyzed by Omega $16 \mathrm{ic}$ Chemiluminescence Imaging System.

\section{TNF- $\alpha$ and $\mathrm{PGE}_{2}$ Assay}

The amount of TNF- $\alpha$ in the culture medium was determined $24 \mathrm{~h}$ after treatment with a rat TNF- $\alpha$ enzyme-linked immunosorbent assay (ELISA) kit (BioSource International, USA). The content of TNF- $\alpha$ in rat brain tissue extracts was also determined by TNF- $\alpha$ ELISA kit. The production of $\mathrm{PGE}_{2}$ in microglia cultures was evaluated $24 \mathrm{~h}$ after treatment with a $\mathrm{PGE}_{2}$ radioimmumoassay kit purchased from Blood Research Institute (Soochow University, China).

\section{Detection of Mitochondrial Membrane Potential}

Microglial mitochondrial membrane potential was detected with the fluorescent probe JC-1 (Molecular Probes, USA), which exists predominantly in monomeric form when cells with depolarized mitochondria and display fluoresced green at $490 \mathrm{~nm}$. Cells with polarized mitochondria predominantly contained JC-1 in aggregate form and show fluoresced reddish-orange. Microglia were incubated with $5 \mu \mathrm{M}$ of JC-1 for $10 \mathrm{~min}$ at $37^{\circ} \mathrm{C}$, washed, and placed on a thermostatted stage at $37^{\circ} \mathrm{C}$. Fluorescent images were visualized by a Nikcon Optical TE2000-S inverted fluorescence microscope with excitation at $490 \mathrm{~nm}$ and emission at $>520 \mathrm{~nm}$. Acquired signal was analyzed with image-analysis software (Simple PCI). A minimum of six fields was selected and average intensity for each region was quantified. The ratio of J-aggregate to JC-1 monomer intensity for each region was calculated. A decrease in this ratio was interpreted as loss of $\Delta \Psi_{\mathrm{m}}$, whereas an increase in the ratio was interpreted as gain in $\Delta \Psi_{\mathrm{m}}$.

\section{Western Blotting}

Cells were collected and homogenized in $200 \mu \mathrm{l}$ lysis buffer. After incubation for $20 \mathrm{~min}$ on ice, cell lysates were centrifuged and protein concentration in the extracts was determined by the Bradford assay. Proteins in cell extracts were denatured with sodium dodecyl sulfate (SDS) sample 
buffer and separated by $10 \%$ SDS-polyacrylamide gel electrophoresis. Proteins were transferred to nitrocellulose membranes using a Bio-Rad miniprotein-III wet transfer unit. The membranes were incubated with 5\% BSA dissolved in Tris-buffered saline with Tween 20 (TBST) (pH 7.5, $10 \mathrm{mM}$ Tris- $\mathrm{HCl}, 150 \mathrm{mM} \mathrm{NaCl}$, and $0.1 \%$ Tween 20) at room temperature for $1 \mathrm{~h}$, washed three times and incubated with different antibodies (p38, phospho-p38, JNK, and phospho-JNK at 1:1000) overnight at $4^{\circ} \mathrm{C}$. The membranes were washed three times with TBST buffer and incubated with the secondary antibody $(1: 1000)$ for $1 \mathrm{~h}$ followed by four washings. Signal detection was performed with an enhanced chemiluminescence kit.

\section{Statistical Analysis}

All values are means \pm SEM. The significance of the difference between control and samples treated with various drugs was determined by one-way ANOVA followed by the post hoc least significant difference test. Differences were considered significant at $P<0.05$.

\section{RESULTS}

Iptakalim Alleviates Rotenone-Induced Rat Behavioral Symptoms and Dopaminergic Neuronal Degeneration in Substantia Nigra

Chronic administration with rotenone $(2.5 \mathrm{mg} / \mathrm{kg} /$ day for 28 days, subcutaneously) induced obvious neurotoxicity in rats, including significant weight loss (data not shown), obvious mortality (40\%), and typical parkinsonian symptoms, such as catalepsy. Rats were received intragastric administration with IPT $(1.5,3.0 \mathrm{mg} / \mathrm{kg} /$ day $)$ or diazoxide $(3.0 \mathrm{mg} / \mathrm{kg} /$ day) for 3 days. On day 4 , IPT or diazoxide was pretreated 1 hour before the injection with rotenone $(2.5 \mathrm{mg} / \mathrm{kg} / \mathrm{day}$, subcutaneously) on a daily basis for 4 weeks. IPT or diazoxide significantly alleviated rotenoneinduced rat weight loss and death (data not shown).

In the rotarod test, vehicle-treated animals learned the task quickly and, after a short training period, were able to remain on the rod at different speed (5-20 r.p.m.). They stepped voluntarily from the hand of the experimenter onto the rotarod, except at very high rotation speeds. Almost all control animals remained on the rod for $300 \mathrm{~s}$ at 5-20 r.p.m. The time-on-the-rod of rotenone-treated rats was significantly reduced with an obvious gradual decrease in timeon-the-rod as rod speed increased. Rats pretreated with IPT or diazoxide had a longer length of time-on-the-rod compared with the rotenone-treated rats (Figure 1a). Using the catalepsy test, rotenone-treated rats showed prolonged latency time compared to that in the vehicle-treated control group. The latency time of rats pretreated with IPT (1.5, $3.0 \mathrm{mg} / \mathrm{kg} /$ day $)$ or diazoxide $(3.0 \mathrm{mg} / \mathrm{kg} /$ day $)$ decreased by 91,78 , and $87 \%$, respectively (Figure $1 \mathrm{~b}$ ).

Furthermore, stereological count of TH-immunoreactive (TH-ir) cells in substantia nigra was used to explore the effects of IPT on rotenone-induced degeneration of dopaminergic neurons. Chronic treatment with rotenone induced a dramatic loss of dopaminergic neurons by $68.1 \%$
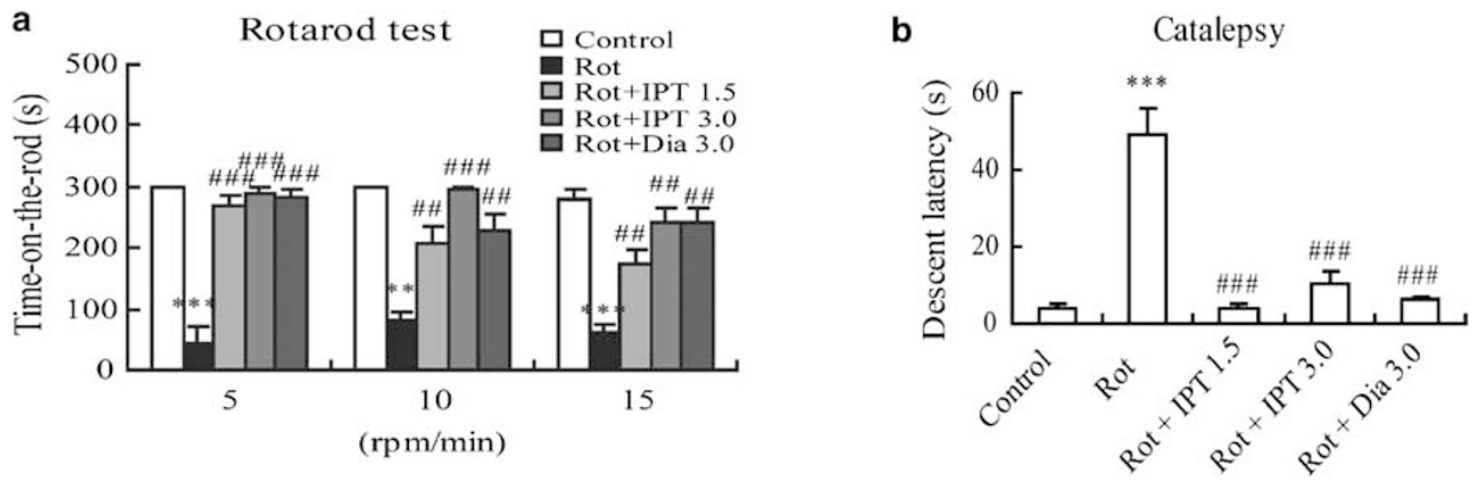
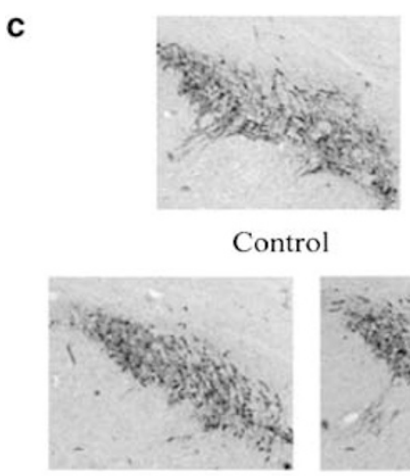

Rot + IPT 1.5

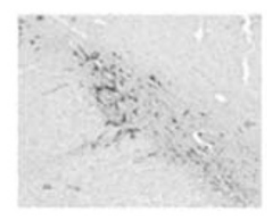

Rot

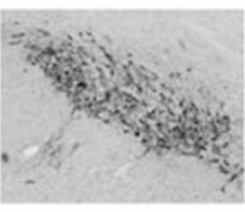

Rot + IPT 3.0

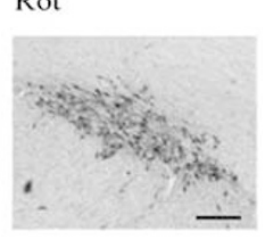

Rot + Dia 3.0

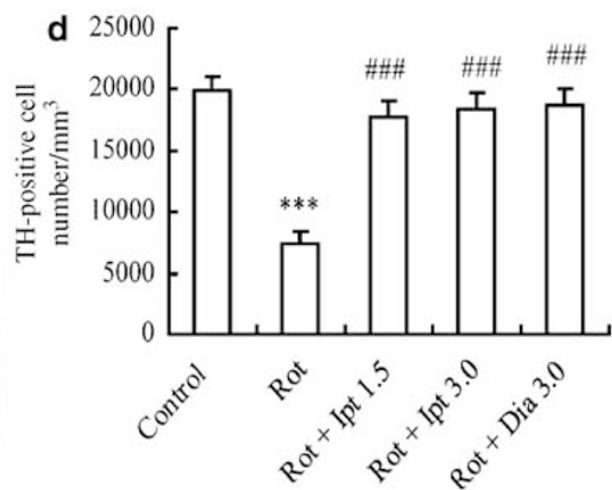

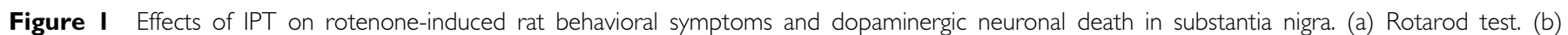

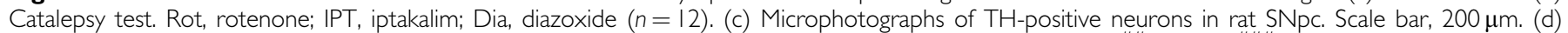

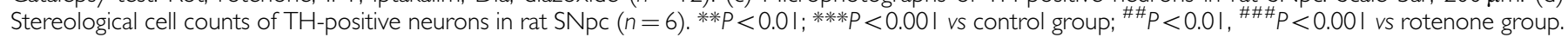
Data are presented as means \pm SEM. 
compared with control group $(P<0.01)$ indicated by obviously reduced number of TH-ir neurons. IPT (1.5, $3.0 \mathrm{mg} / \mathrm{kg} /$ day $)$ or diazoxide $(3.0 \mathrm{mg} / \mathrm{kg} /$ day $)$ remarkably alleviated rotenone-induced dopaminergic neurons degeneration by $22.2,15.8$, and $19.7 \%$, respectively (Figure $1 \mathrm{c}$ and d).

\section{Iptakalim Inhibits Rotenone-Induced Microglial Activation in Rat Substantia Nigra}

To explore the effects of IPT on microglial activation, immunostaining was used to detect OX-42 and ED1 markers for microglia. As shown in Figure 2, chronic administration with rotenone $(2.5 \mathrm{mg} / \mathrm{kg} /$ day, s.c.) for 4 weeks could induce notable microglial activation in rat substantia nigra indicated by large number of OX-42-ir and ED1-ir cells, which had larger cell body, poorly ramified short, and thick processes. IPT $(1.5,3.0 \mathrm{mg} / \mathrm{kg} /$ day $)$ or diazoxide
$(3.0 \mathrm{mg} / \mathrm{kg} /$ day $)$ pretreatment resulted in significant suppression of microglial activation in rat substantia nigra, indicated by few OX-42-ir and ED1-ir cell number, small cell body, ramified, and thin processes (Figure 2). These results suggest that IPT could inhibit rotenone-induced microglial activation, and this inhibitory effect may be implicated in the neuroprotective effects of IPT.

\section{Iptakalim Inhibits Rotenone-Induced Production of TNF- $\alpha$ and COX-2 in Rat Substantia Nigra}

As microglia-mediated neuroinflammation is mainly owing to the excessive proinflammatory and cytotoxic factors from activated microglia and their downstream signaling cascades, the mRNA levels of proinflammatory factors were detected. With GAPDH as an internal control, the results of semiquantitative reverse transcription-polymerase chain

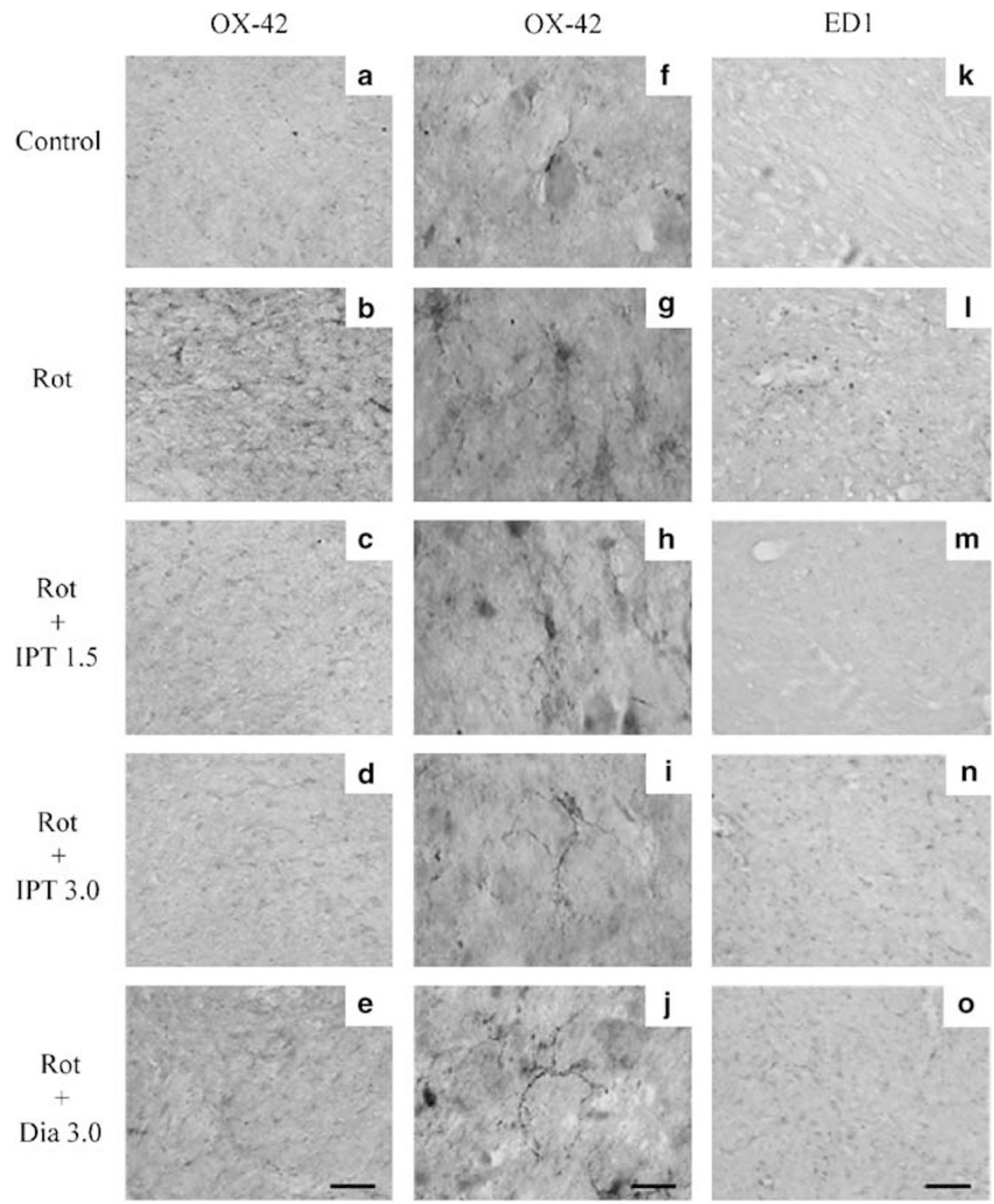

Figure 2 Effects of IPT on rotenone-induced microglial activation in rat substantia nigra. OX-42 and EDI immunostaining were performed to study microglial activation. Rats treated with vehicle showed minimal microglial activation in SNpc $(a, f, k)$ indicated by few OX-42-ir and EDI-ir cell number and small cell body with ramified and thin processes (f). In rotenone-treated rats, there were numerous OX-42-ir and EDI-ir cells in SNpc (b, g, I), which had larger cell body, poorly ramified short and thick processes (g). Rats pretreated with IPT ( 1.5 or $3.0 \mathrm{mg} / \mathrm{kg} /$ day) or diazoxide ( $1.5 \mathrm{mg} / \mathrm{kg} /$ day) reduced the number of rotenone-induced OX-42- and EDI-positive cells, which was similar to those seen in vehicle-treated rats. Scale bar: a-e, I00 $\mu \mathrm{m}$; $\mathrm{f}-\mathrm{j}, 20 \mu \mathrm{m}$; $k-0,100 \mu \mathrm{m}$. 
reaction showed obvious elevation of TNF- $\alpha$ and COX-2 mRNA expression in rotenone-treated rat substantia nigra. The mRNA level of TNF- $\alpha$ was increased by about 3.1 $(P<0.01 v s$ control $)$ times that of control group. Similarly, the level of COX-2 mRNA was upregulated by 2.1 times $(P<0.01 v s$ control $)$. IPT $(1.5,3.0 \mathrm{mg} / \mathrm{kg} /$ day $)$ or diazoxide $(3.0 \mathrm{mg} / \mathrm{kg} /$ day) could significantly downregulate mRNA levels of TNF- $\alpha$ and COX-2 in rotenone-treated rat substantia nigra (Figure $3 \mathrm{a}$ and $\mathrm{b}$ ). Additionally, the content of TNF- $\alpha$ in rat brain tissue extracts of substantia nigra was determined. The results showed that chronic treatment with rotenone $(2.5 \mathrm{mg} / \mathrm{kg} /$ day $)$ significantly increased TNF- $\alpha$ content in substantia nigra (297\% of control). However, IPT $(1.5,3.0 \mathrm{mg} / \mathrm{kg} /$ day $)$ or diazoxide $(1.5 \mathrm{mg} / \mathrm{kg} /$ day $)$ could attenuate the content of TNF- $\alpha$ in substantia nigra compared with that of rotenone-treated rats (Figure $3 \mathrm{c}$ ). Similar results were observed in rat striatum (data not shown). These results further suggest that IPT could obviously suppress rotenone-induced microglial activation and neuroinflammation, which may result in the alleviation of dopaminergic neuronal degeneration.

\section{Iptakalim Decreases Rotenone-Induced Production of TNF- $\alpha$ and PGE2 from Rat Primary Cultured Microglia}

To further investigate the inhibitory effect of IPT on microglial activation, rat primary cultured microglia were cultured for in vitro study. As shown in Figure 4, incubation with rotenone $(10 \mathrm{nM})$ for $24 \mathrm{~h}$ significantly increased the production of TNF- $\alpha$ and $\mathrm{PGE}_{2}(P<0.01$ vs control). Pretreatment with IPT $(0.01,0.1,1,10,100 \mu \mathrm{M}) 20 \mathrm{~min}$ before addition of rotenone produced a concentrationdependent decrease in TNF- $\alpha$ production with a minimum effective dose of $0.1 \mu \mathrm{M}$ (Figure $4 \mathrm{a}$ ). Similarly, IPT $(0.01,0.1$, $1,10,100 \mu \mathrm{M})$ also decreased the $\mathrm{PGE}_{2}$ production at a minimum effective dose of $0.1 \mu \mathrm{M}$ (Figure $4 \mathrm{~b}$ ).

IPT $(10 \mu \mathrm{M})$ alone failed to affect TNF- $\alpha$ and $\mathrm{PGE}_{2}$ production from untreated microglial (data not shown). However, IPT $(10 \mu \mathrm{M})$ reduced rotenone-induced TNF- $\alpha$ production up to $35.1 \%$ along with the reduced production of $\mathrm{PGE}_{2}$ up to $40.6 \%$ (Figure $4 \mathrm{c}$ and d). Preincubation with $K_{\text {ATP }}$ channel blocker glibenclamide $(10 \mu \mathrm{M})$ or selective mito $K_{\mathrm{ATP}}$ channel blocker 5-HD $(250 \mu \mathrm{M})$ for 20 min could reverse these inhibitory effects of IPT. These results indicated that IPT could inhibit rotenone-induced production of proinflammatory factors from microglia through opening mito $K_{\mathrm{ATP}}$ channels.

\section{Iptakalim Inhibits Rotenone-Induced Microglial Activation In Vitro}

The amoeboid morphological changes and increased ED1 expression were hallmarks of microglial activation. Typically, 'resting' microglia are characterized by ramified and either bipolar or unipolar processes with low level of ED1 expression, whereas activated microglia become round with enlarged and amoeboid cell bodies, accompanied by increased expression of ED1. After incubation with $10 \mathrm{nM}$ rotenone for $24 \mathrm{~h}$, most microglia were activated, indicated by dramatic amoeboid morphological changes (Figure 5a) and significant upregulation of ED1 expression (Figure 5b and c). Preincubation with IPT $(10 \mu \mathrm{M})$ or diazoxide $(100 \mu \mathrm{M})$ for $20 \mathrm{~min}$ obviously attenuated rotenone-induced morphological alterations. Meanwhile, ED1 expression was decreased by 62.3 and $65.6 \%$, respectively. Preincubation with the selective mito $K_{\mathrm{ATP}}$ channel blocker 5 -HD $(250 \mu \mathrm{M})$ could reverse the inhibitory effects of IPT and diazoxide. Neither $10 \mu \mathrm{M}$ IPT nor $100 \mu \mathrm{M}$ diazoxide alone induced obvious microglial activation (data not shown). These results demonstrate that IPT could inhibit rotenoneinduced microglial activation in vitro through opening mito $K_{\text {ATP }}$ channels.

\section{Iptakalim Prevents Rotenone-Induced Mitochondrial Membrane Potential Loss in Microglia}

As mitochondrial membrane potential $\left(\Delta \Psi_{\mathrm{m}}\right)$ change has been demonstrated to be involved in microglial activation a
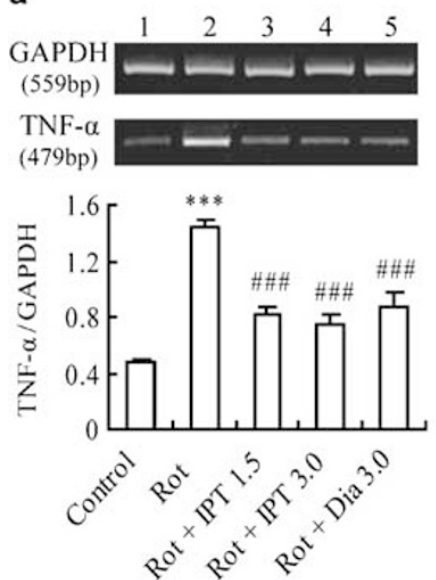

b
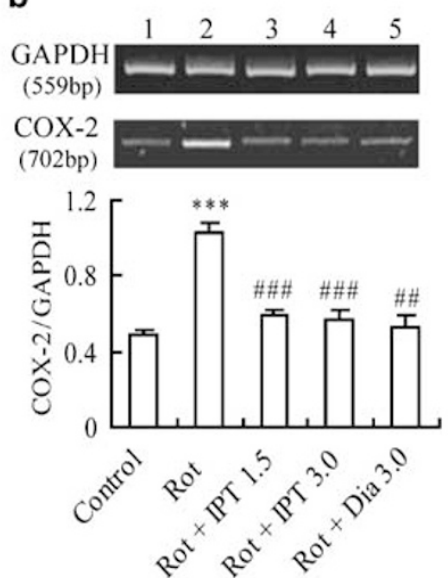

C

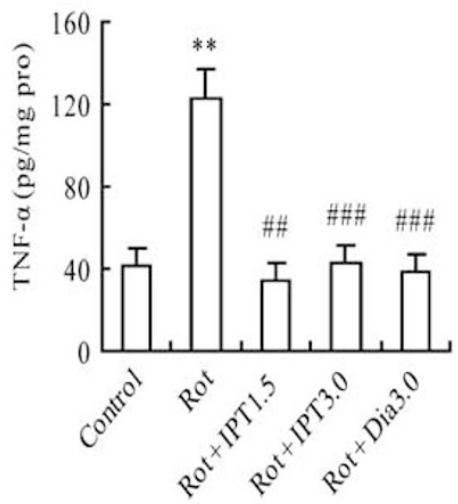

Figure 3 Effects of IPT on rotenone-induced production of proinflammatory factors in rat substantia nigra. IPT downregulated the mRNA levels of TNF- $\alpha$ (a) and COX-2 (b) in substantia nigra. Rats were grouped as below: Group I: control, Group2: rotenone (2.5 mg/kg/day), Group3: rotenone (2.5 mg/kg/day) + IPT ( $1.5 \mathrm{mg} / \mathrm{kg} /$ day), Group4: rotenone $(2.5 \mathrm{mg} / \mathrm{kg} / \mathrm{day})+$ IPT $(3.0 \mathrm{mg} / \mathrm{kg} /$ day), Group5: rotenone $(2.5 \mathrm{mg} / \mathrm{kg} / \mathrm{day})+$ diazoxide (I.5 mg/kg/day). (c) IPT

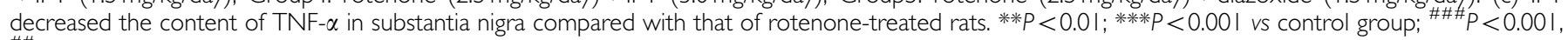
${ }^{\# \#} P<0.0$ I vs rotenone group. Data are presented as mean $\pm S E M, n=4$. 
a

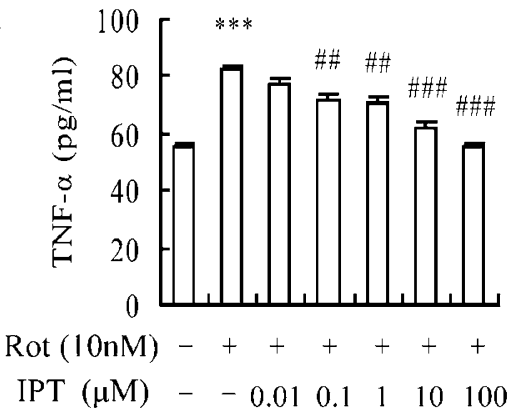

b

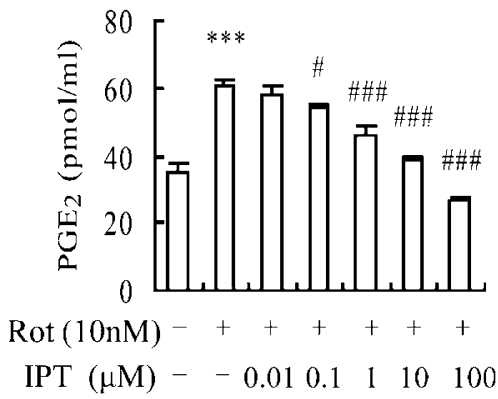

C

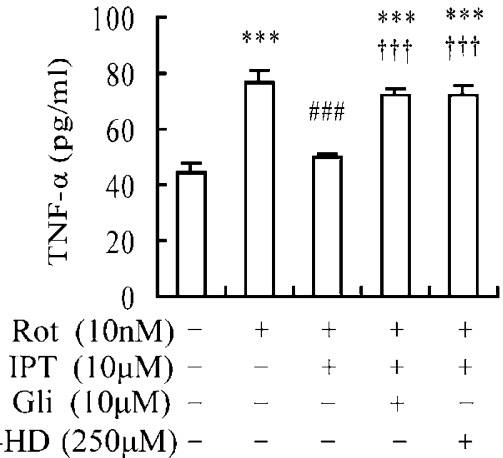

d

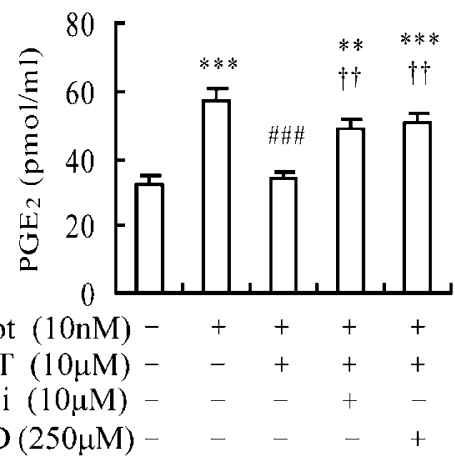

Figure 4 Effects of IPT on rotenone-induced production of TNF- $\alpha(a, c)$ and PGE 2 (b, d) from microglia. Rot: rotenone. IPT: iptakalim. Gli, glibenclamide.

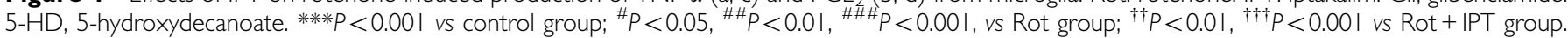
Data are presented as the mean \pm SEM of four independent experiments.

and production of proinflammatory factors, molecular probe JC-1 was used to detect the effect of IPT on rotenone-induced microglial mitochondrial membrane potential variation, which may be implicated in the neuroprotection of IPT. As shown in Figure 6, after exposure to rotenone $(10 \mathrm{nM})$ for $30 \mathrm{~min}$, most microglia displayed a loss or collapse of $\Delta \Psi_{\mathrm{m}}$ indicated by fluorescence of JC-1 shifted from red-orange to greenish-yellow. Pretreatment with IPT $(10 \mu \mathrm{M})$ prevented the loss of $\Delta \Psi_{\mathrm{m}}$ in microglia. Furthermore, 5-HD $(250 \mu \mathrm{M})$ could abolish the preventive effects of IPT. These data indicate that IPT may prevent rotenone-induced mitochondrial membrane depolarization through activating mito $K_{\mathrm{ATP}}$ channels.

\section{Iptakalim Suppresses Rotenone-Induced p38/JNK MPAK Activation in Microglia}

Mitogen-activated protein kinases (MAPKs), the predominant signaling transduction pathway responsible for synthesis and production of proinflammatory factors in microglia, can be regulated by mitochondrial function (Akundi et al, 2005; Ciallella et al, 2005; Lund et al, 2005; Waetzig et al, 2005). In the present study, microglia were treated with rotenone $(10 \mathrm{nM})$ for different intervals of time to determine the involvement of MAPK after rotenone stimulation. Treatment with rotenone led to a rapid and transient phosphorylation of both p38 and JNK MAPK, indicating activation of $\mathrm{p} 38$ and JNK with the peak levels of phosphorylated p38 and JNK occurring at 30 min (Figure 7a and b). Phosphorylated p38 sustained up to $2 \mathrm{~h}$ after rotenone addition, but JNK phosphorylation was still detectable at $4 \mathrm{~h}$. These data suggest that both p38 and
JNK MAPK are activated in response to rotenone stimulation in microglia.

Next, we explored whether IPT could regulate rotenoneinduced $\mathrm{p} 38 / \mathrm{JNK}$ MAPK phosphorylation at $30 \mathrm{~min}$ time point. Pretreatment with $10 \mu \mathrm{M}$ IPT suppressed rotenoneinduced increase of phospho-p38 and phospho-JNK by over 50 and $64 \%$, respectively (Figure $7 \mathrm{c}$ and $\mathrm{d}$ ). Moreover, these suppressive effects of IPT were reversed by mito $K_{\text {ATP }}$ channel blocker 5-HD $(250 \mu \mathrm{M})$. These data suggest that IPT may inhibit rotenone-induced microglial activation through regulating mitochondrial function and the downstream MAPK signaling transduction.

\section{DISCUSSION}

Microglial activation has been demonstrated to be an early sign that often precedes and triggers neuronal death in chronic neurodegenerative diseases, such as in PD (Gao et al, 2003a, b; Block and Hong, 2005; Minghetti, 2005). Chronic administration of rotenone reproduced key features of PD in rodents, and microglial activation might be the predominant mechanism for rotenone-induced dopaminergic neuronal degeneration (Alam and Schmidt, 2002; Bonetta, 2002; Gao et al, 2002, 2003a; Perier et al, 2003; Sherer et al, 2003; Hirsch et al, 2005). Therefore, inhibition of microglial activation and subsequent neuroinflammation may offer prospective clinical therapeutic benefit for PD and other neuroinflammation-related neurodegenerative disorders.

IPT, a $K_{\text {ATP }}$ channel opener that can freely cross the blood-brain barrier, has been demonstrated to exert significant neuroprotective effects, such as promoting 
a

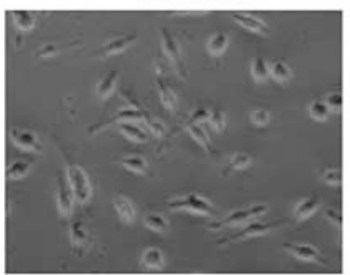

Rot

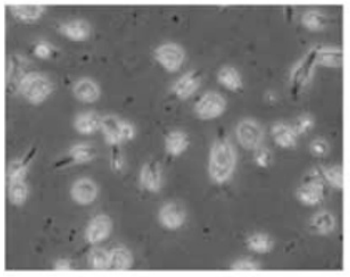

b

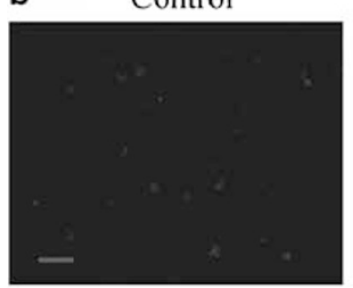

Rot

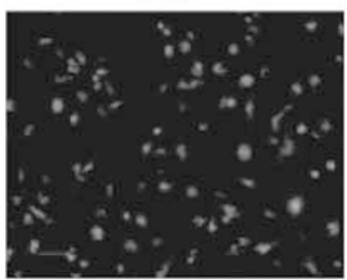

Rot + IPT

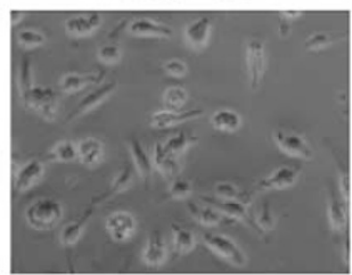

Rot + IPT + 5-HD

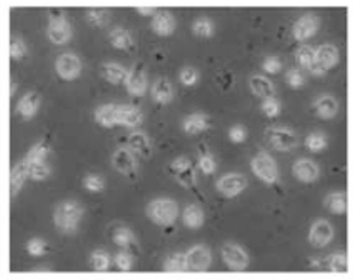

Rot + IPT

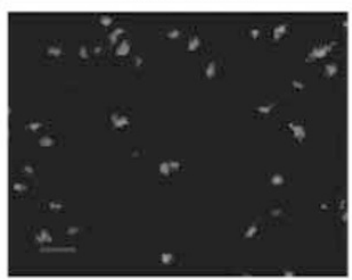

Rot + IPT + 5-HD

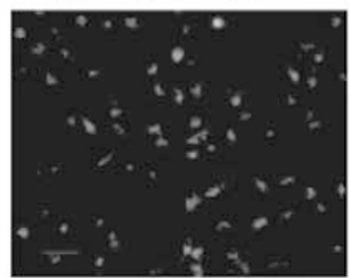

Rot + Dia

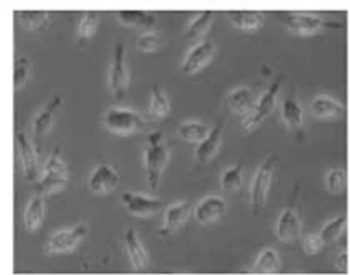

Rot + Dia + 5-HD
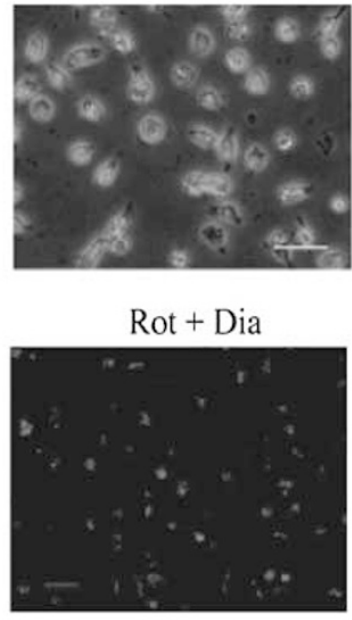

Rot + Dia + 5-HD

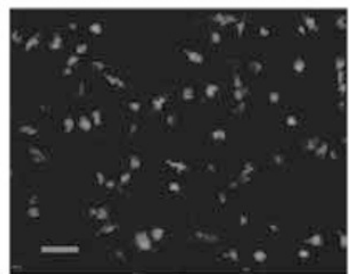

C

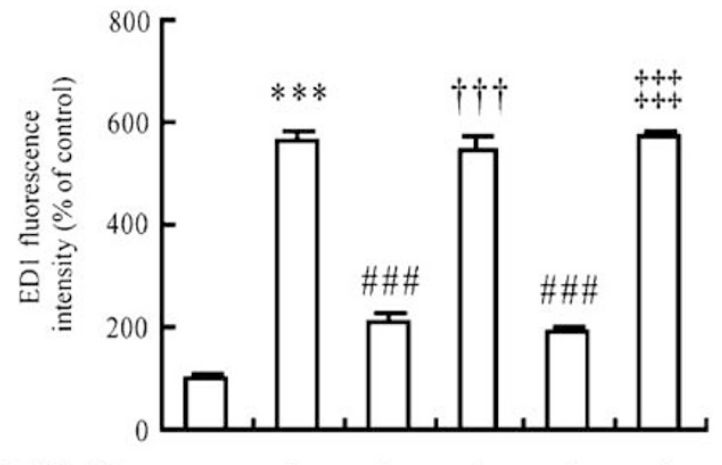

$\begin{array}{ccccccc}\text { Rot }(10 \mathrm{nM}) & - & + & + & + & + & + \\ \operatorname{IPT}(10 \mu \mathrm{M}) & - & - & + & + & - & - \\ \operatorname{Dia}(100 \mu \mathrm{M}) & - & - & - & - & + & + \\ 5-\mathrm{HD}(250 \mu \mathrm{M}) & - & - & - & + & - & +\end{array}$

Figure 5 Effects of IPT on rotenone-induced microglial activation. (a) Morphological changes of microglia. (b) Microglia were stained with the activated microglial marker EDI. (c) Data for the fluorescence intensity of EDI-positive microglia. ${ }^{* * *} P<0.001$ vs control group; ${ }^{\# \#} P<0.00$ I vs Rot group; ${ }^{\dagger \dagger \dagger} P<0.00$ I vs Rot + IPT group; ${ }^{\ddagger \ddagger \ddagger} P<0.00$ I vs Rot + Dia (diazoxide) group. Data are presented as the mean \pm SEM of four independent experiments. Scale bar, $50 \mu \mathrm{m}$.

behavioral recovery, protecting neurons against necrosis and apoptosis in different animal models of stroke, PD, as well as in cultured cells (Wang et al, 2004, 2005, 2006; $\mathrm{Hu}$ et al, 2005; Yang et al, 2006). Additionally, our previous study showed that IPT exhibited potent neuroprotective effects against rotenone-induced neurotoxicity by inhibiting iNOS expression and activity (Yang et al, 2005, 2006). In the present study, systematic administration with IPT could 

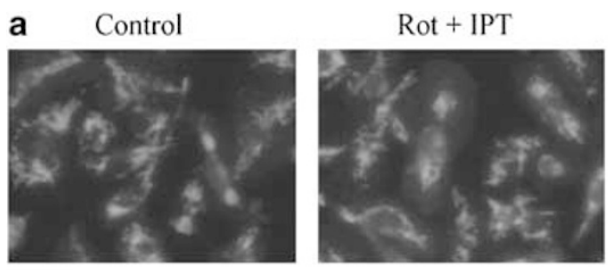

Rot

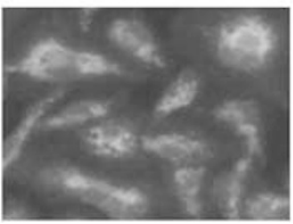

Rot + IPT + 5-HD

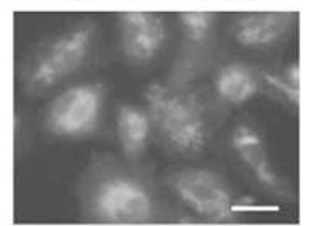

b

Fluorescence ratio
J-aggregate: JC-1 monomer

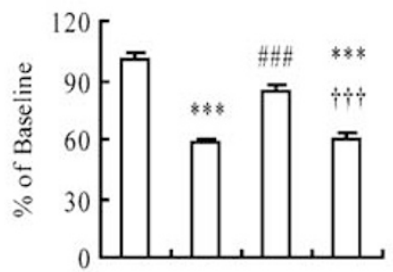

Rot $(10 \mathrm{nM})-+++$

IPT $(10 \mu \mathrm{M})-{ }_{-}+$

$5-\mathrm{HD}(250 \mu \mathrm{M})-\quad-\quad-\quad+$

Figure 6 Effects of IPT on rotenone-induced mitochondrial depolarization in microglia. (a) JC-I fluorescence images of mitochondria. Scale bar, $25 \mu$ m. (b) Quantification of mitochondrial membrane potential expressed as a ratio of J-aggregate to JC-I monomer fluorescence intensity. $* * * * P<0.00$ I vs control groups; ${ }^{\# \#} P<0.00$ I, vs Rot group; ${ }^{\dagger \dagger} P<0.00$ I vs Rot + IPT group. Data are presented as the mean \pm SEM of four independent experiments.

a

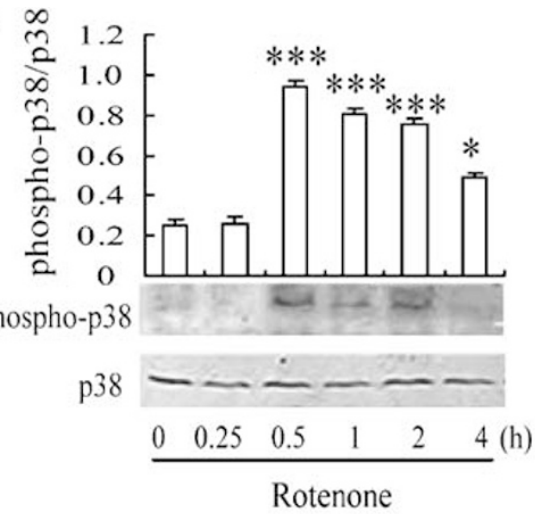

C

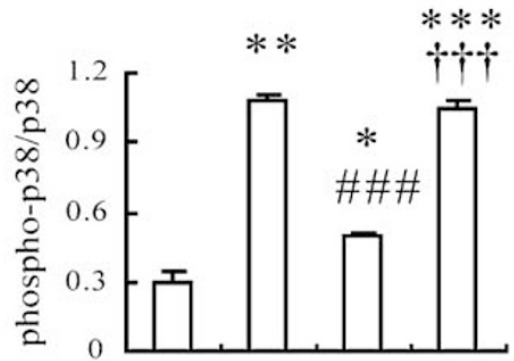

phospho-p38

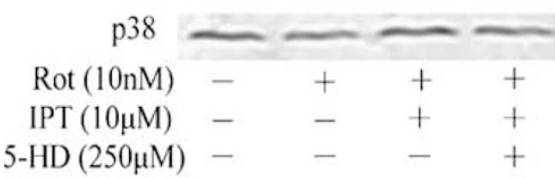

b

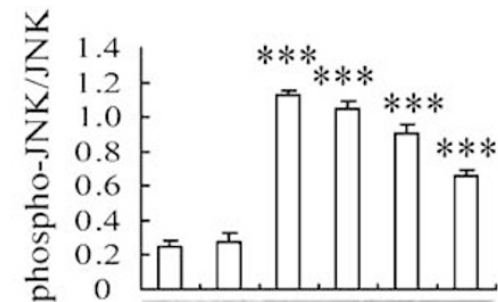

phospho-JNK

JNK

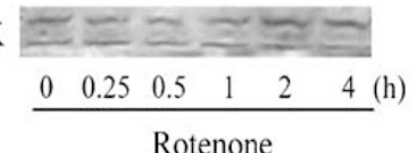

d

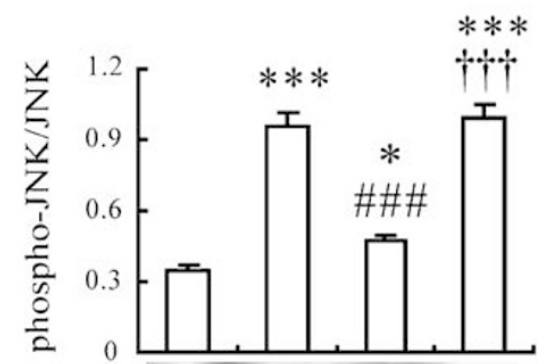

phospho-JNK

JNK

Rot (10nM)

$++\frac{1}{-1}+$

5 - $\mathrm{HD}(250 \mu \mathrm{M}) \quad-\quad-\quad-\quad+\quad+$

Figure 7 Effects of IPT on rotenone-induced p38/JNK MAPK activation in microglia. Rotenone increased p38/JNK MAPK phosphorylation, indicative of p38/JNK MAPK activation. Microglia were treated with $10 \mathrm{~nm}$ rotenone for indicated times $(\mathrm{a}, \mathrm{b})$. Pretreatment with $10 \mu \mathrm{M}$ IPT suppressed rotenoneinduced p38/JNK phosphorylation (c, d). 30 min after treatment, microglia were harvested and phosphorylated p38/JNK MAPK were analyzed. Lower: Representative blots are shown. Upper: Densitometric analysis of the phosphorylated forms of $p 38 / J N K M A P K$. $* P<0.05$, *** $P<0.0$ I. **** $P<0.00$ I vs

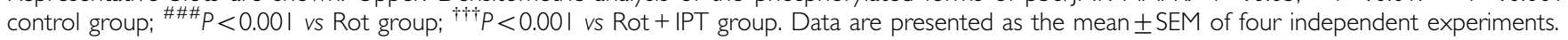

alleviate rotenone-induced dopaminergic neuron loss along with the inhibition of microglial activation and production of proinflammatory factors in substantia nigra. Our in vitro studies further confirmed that IPT could suppress rotenone-induced microglial activation and production of proinflammatory factors. Because microglial activation is essential for upregulation of proinflammatory factors in substantia nigra (Arimoto and Bing, 2003), our findings suggest that the neuroprotection exerted by IPT is likely owing to inhibition of microglial activation, which may in turn slow the progressive dopaminergic neuronal degeneration. 
In the present study, it was demonstrated that the inhibitory effects of IPT on microglial activation and production of proinflammatory factors were reversed by selective mito $K_{\mathrm{ATP}}$ channel blocker $5-\mathrm{HD}$, which suggests that IPT may suppress microglial activation through opening mito $K_{\mathrm{ATP}}$ channels in microglia. It is well documented that $K_{\mathrm{ATP}}$ channel, especially mito $K_{\mathrm{ATP}}$ channel, may be a novel protective target for neuron and astrocyte (Yamauchi et al, 2003; Busija et al, 2004). Accumulating evidence has showed that the amount of mito $K_{\mathrm{ATP}}$ channels located in brain cells is at least sixfold higher than that in heart cells (Bajgar et al, 2001; Lacza et al, $2003)$, indicating an essential role of mito $K_{\mathrm{ATP}}$ channel in the physiology and pathology of CNS. Consistent with the results of our present study, Farkas et al $(2005 \mathrm{a}, \mathrm{b})$ reported that mito $K_{\mathrm{ATP}}$ channel opener diazoxide could alleviate ischemia-related white matter injury and learning dysfunction by suppressing microglial activation. Thus, mito $K_{\mathrm{ATP}}$ channels may be an important molecular target for inhibiting microglia-mediated neuroinflammation and for treating neuroinflammation-related neurodegenerative disorders. Although Liss et al (2005) reported that genetic inactivation of Kir6.2 resulted in a selective rescue of substantia nigra dopaminergic neurons in a MPTP model of dopaminergic degeneration, there were a large number of evidences for benefits afforded by activating mito $K_{\mathrm{ATP}}$ in ischemia and degeneration (Yamauchi et al, 2003; Busija et al, 2004; Farkas et al, 2005a, b). This discrepancy may be owing to the difference between the opening of mito $K_{\mathrm{ATP}}$ channels and genetic inactivation of Kir6.2. In addition, the $K_{\text {ATP }}$ channels in dopaminergic neurons composed of Kir6.2 and SUR1 subunits display a promotion of neurodegenerative process in PD (Liss et al, 2005; Deutch and Winder, 2006), but it is worth noting that the composing subunits of $K_{\text {ATP }}$ channels in microglia differ from that of dopaminergic neurons and may play a distinct role in the initiation and process in the degenerative diseases.

Increasing evidence has showed that mito $K_{\mathrm{ATP}}$ channels play an important role in maintaining mitochondrial function by regulating intracellular signal transduction, mitochondrial volume, and calcium homeostasis, which may be involved in cell survival and death (Busija et al, 2004; Ardehali and O'Rourke, 2005; Rodrigo and Standen, 2005; Nichols, 2006). However, the exact mechanism underlying the inhibition of microglial activation by opening mito $K_{\mathrm{ATP}}$ channels remains unknown. Mitochondrial membrane potential and the downstream MAPKs have been demonstrated to regulate microglial activation and the production of proinflammatory factors from microglia (Akundi et al, 2005; Ciallella et al, 2005; Lund et al, 2005; Waetzig et al, 2005). The inhibitory effects of IPT on rotenone-induced $\Delta \Psi_{\mathrm{m}}$ loss and $\mathrm{p} 38 / \mathrm{JNK}$ MAPK activation were abolished by addition of 5-HD, indicating IPT might regulate mitochondrial function and MAPKs pathways through opening mito $K_{\mathrm{ATP}}$ channels. Recently, ROS generated in the mitochondrial respiratory chain is considered as an important mediator of signal transduction in ischemic preconditioning (Otani, 2004), which activates p38 and JNK MAPK through the activation of upstream signaling MKK. Activation of mito $K_{\mathrm{ATP}}$ channel increases production of protective ROS during preconditioning and decreases the levels of harmful ROS produced during reperfusion
(O'Rourke, 2004). So we propose that opening mito $K_{\mathrm{ATP}}$ channel by IPT may inhibit the rotenone-induced production of proinflammatory factors from microglia through suppression of mitochondria-derived ROS and downstream MAPKs phosphorylation.

In addition, our results showed that the activity of IPT was compared to the well-characterized mito $K_{\mathrm{ATP}}$ channel opener diazoxide. Diazoxide was originally developed as an antihypertensive agent, but was found to induce hyperglycemia by reducing insulin secretion. The clinical use of diazoxide has been hampered by its lack of potency and selectivity giving rise to side effects (Hansen, 2006). In contrast, IPT possesses several advantages, such as free penetration through the blood-brain barrier and low-toxic side effects during systemic administration (Wu et al, 2005). Therefore, IPT is a promising compound that may protect neurons against a variety of neurodegenerative diseases.

In conclusion, we reported first that IPT significantly inhibited rotenone-induced microglial activation and subsequent production of proinflammatory factors through activating mito $K_{\mathrm{ATP}}$ channels whereby alleviated the dopaminergic neuronal degeneration. These data strongly support the notion that inhibition of microglial activation by opening mito $K_{\mathrm{ATP}}$ channels may provide a new therapeutic strategy for neuroinflammation-related disorders. IPT, a $K_{\mathrm{ATP}}$ channel opener that can pass through the blood-brain barrier and has few effects on peripheral blood pressure, might be developed as a potential therapeutic agent for PD and other neuroinflammation-related neurodegenerative diseases.

\section{ACKNOWLEDGEMENTS}

This study was partly supported by a grant from the National Natural Science Foundation of China (Nos. 30625038, 30572172, and 30600758), a grant from the Key Project of Natural Science Foundation of Jiangsu Educational Department (Nos. 05KJA31014 and 06KJA31029), a grant from the Key Project of Jiangsu Health Department (No. K200501), and a Specialized Research Fund for the Doctoral Program of Higher Education of China (No. 20040312004).

\section{DISCLOSURE/CONFLICTS OF INTEREST}

There is no duality of interest that we should disclose.

\section{REFERENCES}

Akundi RS, Candelario-Jalil E, Hess S, Hull M, Lieb K, GebickeHaerter PJ et al (2005). Signal transduction pathways regulating cyclooxygenase-2 in lipopolysaccharide-activated primary rat microglia. Glia 51: 199-208.

Alam M, Schmidt WJ (2002). Rotenone destroys dopaminergic neurons and induces parkinsonian symptoms in rats. Behav Brain Res 136: 317-324.

Ardehali H, O’Rourke B (2005). Mitochondrial K(ATP) channels in cell survival and death. J Mol Cell Cardiol 39: 7-16.

Arimoto T, Bing G (2003). Up-regulation of inducible nitric oxide synthase in the substantia nigra by lipopolysaccharide causes microglial activation and neurodegeneration. Neurobiol Dis 12: $35-45$. 
Bajgar R, Seetharaman S, Kowaltowski AJ, Garlid KD, Paucek P (2001). Identification and properties of a novel intracellular (mitochondrial) ATP-sensitive potassium channel in brain. J Biol Chem 276: 33369-33374.

Block ML, Hong JS (2005). Microglial and inflammation-mediated neurodegeneration: multiple triggers with a common mechanism. Prog Neurobiol 76: 77-98.

Bonetta L (2002). Pesticide-Parkinson link explored. Nat Med 8: 1050.

Busija DW, Lacza Z, Rajapakse N, Shimizu K, Kis B, Bari F et al (2004). Targeting mitochondrial ATP-sensitive potassium channels - a novel approach to neuroprotection. Brain Res Rev 46: 282-294.

Ciallella JR, Saporito M, Lund S, Leist M, Hasseldam H, McGann N et al (2005). CEP-11004, an inhibitor of the SAPK/JNK pathway, reduces TNF-alpha release from lipopolysaccharide-treated cells and mice. Eur J Pharmacol 515: 179-187.

Deutch AY, Winder DG (2006). A channel to neurodegeneration. Nat Med 1: 17-18.

Farkas E, Annahazi A, Institoris A, Mihaly A, Luiten PG, Bari F (2005a). Diazoxide and dimethyl sulphoxide alleviate experimental cerebral hypoperfusion-induced white matter injury in the rat brain. Neurosci Lett 373: 195-199.

Farkas E, Timmer NM, Domoki F, Mihaly A, Luiten PG, Bari F (2005b). Post-ischemic administration of diazoxide attenuates long-term microglial activation in the rat brain after permanent carotid artery occlusion. Neurosci Lett 387: 168-172.

Gao HM, Hong JS, Zhang W, Liu B (2002). Distinct role for microglial in rotenone-induced degeneration of dopaminergic neurons. J Neurosci 22: 782-790.

Gao HM, Liu B, Hong JS (2003a). Critical role for microglial NADPH oxidase in rotenone-induced degeneration of dopaminergic neurons. J Neurosci 23: 6181-6187.

Gao HM, Liu B, Zhang W, Hong JS (2003b). Novel antiinflammatory therapy for Parkinson's disease. Trends Pharmacol Sci 24: 395-401.

Hansen JB (2006). Towards selective Kir6.2/SUR1 potassium channel openers, medicinal chemistry and therapeutic perspectives. Curr Med Chem 13: 361-376.

Helmuth L (2000). Pesticide causes Parkinson's in rats. Science 290: 1068.

Herrera AJ, Tomas-Camardiel M, Venero JL, Cano J, Machado A (2005). Inflammatory process as a determinant factor for the degeneration of substantia nigra dopaminergic neurons. J Neural Transm 112: 111-119.

Hirsch EC, Hunot S, Hartmann A (2005). Neuroinflammatory processes in Parkinson's disease. Parkinsonism Relat Disord 11(Suppl 1): S9-S15.

Hu LF, Wang S, Shi XR, Yao HH, Sun YH, Ding JH et al (2005). ATP-sensitive potassium channel opener iptakalim protected against the cytotoxicity of MPP+ on SH-SY5Y cells by decreasing extracellular glutamate level. J Neurochem 94: 1570-1579.

Jenner P (2001). Parkinson's disease, pesticides and mitochondrial dysfunction. Trends Neurosci 24: 245-247.

Lacza Z, Snipes JA, Kis B, Szabo C, Grover G, Busija DW (2003). Investigation of the subunit composition and the pharmacology of the mitochondrial ATP-dependent $\mathrm{K}^{+}$channel in the brain. Brain Res 994: 27-36.

Liss B, Haeckel O, Wildmann J, Miki T, Seino S, Roeper J (2005). K-ATP channels promote the differential degeneration of dopaminergic midbrain neurons. Nat Neurosci 8: 1742-1751.

Liss B, Roeper J (2001). Molecular physiology of neuronal K-ATP channels (review). Mol Membr Biol 18: 117-127.

Liu X, Wu JY, Zhou F, Sun XL, Yao HH, Yang Y et al (2006). The regulation of rotenone-induced inflammatory factor production by ATP-sensitive potassium channel expressed in BV-2 cells. Neurosci Lett 394: 131-135.

Lund S, Porzgen P, Mortensen AL, Hasseldam H, Bozyczko-Coyne $\mathrm{D}$, Morath $\mathrm{S}$ et al (2005). Inhibition of microglial inflammation by the MLK inhibitor CEP-1347. J Neurochem 92: 1439-1451.

Minghetti L (2005). Role of inflammation in neurodegenerative diseases. Curr Opin Neurol 18: 315-321.

Nichols CG (2006). KATP channels as molecular sensors of cellular metabolism. Nature 440: 470-476.

O’Rourke B (2004). Evidence for mitochondrial K+ channels and their role in cardioprotection. Circ Res 94: 420-432.

Otani H (2004). Reactive oxygen species as mediators of signal transduction in ischemic preconditioning. Antioxid Redox Signal 6: 449-469.

Paxinos G, Watson C (1997). The Rat Brain in Stereotaxic Coordinates, Compact, 3rd edn. Academic Press: San Diego.

Perier C, Bove J, Vila M, Przedborski S (2003). The rotenone model of Parkinson's disease. Trends Neurosci 26: 345-346.

Rodrigo GC, Standen NB (2005). ATP-sensitive potassium channels. Curr Pharm Des 11: 1915-1940.

Sherer TB, Betarbet R, Kim JH, Greenamyre JT (2003). Selective microglial activation in the rat rotenone model of Parkinson's disease. Neurosci Lett 341: 87-90.

Waetzig V, Czeloth K, Hidding U, Mielke K, Kanzow M, Brecht S et al (2005). c-Jun N-terminal kinases (JNKs) mediate proinflammatory actions of microglia. Glia 50: 235-246.

Wang H, Zhang YL, Tang XC, Feng HS, Hu G (2004). Targeting ischemic stroke with a novel opener of ATP-sensitive potassium channels in the brain. Mol Pharmacol 66: 1160-1168.

Wang S, Hu LF, Yang Y, Ding JH, Hu G (2005). Studies of ATP-sensitive potassium channels on 6-hydroxydopamine and haloperidol rat models of Parkinson's disease: implications for treating Parkinson's disease? Neuropharmacology 48: 984-992.

Wang S, Hu LF, Zhang Y, Sun T, Sun YH, Liu SY et al (2006). Effects of systematic administration of iptakalim on extracellular neurotransmitter levels in the striatum of unilateral 6-hydroxydopamine-lesioned rats. Neuropsychopharmacology 31: 933-940.

West MJ (1993). New stereological methods for counting neurons. Neurobiol Aging 14: 275-285.

Wu J, Wakui M, Wang H, Hu G (2005). Iptakalim hydrochloride and neuronal protection. Curr Neuropharmacol 3: 249-256.

Xie W, Wang H, Ding J, Wang H, Hu G (2005). Anti-proliferating effect of iptakalim, a novel KATP channel opener, in cultured rabbit pulmonary arterial smooth muscle cells. Eur J Pharmacol 511: 81-87.

Yamada K, Inagaki N (2005). Neuroprotection by KATP channels. J Mol Cell Cardiol 38: 945-949.

Yamauchi T, Kashii S, Yasuyoshi H, Zhang S, Honda Y, Akaike A (2003). Mitochondrial ATP-sensitive potassium channel: a novel site for neuroprotection. Invest Ophthalmol Vis Sci 44: 2750-2756.

Yang Y, Liu X, Ding JH, Sun J, Long Y, Wang F et al (2004). Effects of iptakalim on rotenone-induced cytotoxicity and dopamine release from PC12 cells. Neurosci Lett 366: 53-57.

Yang Y, Liu X, Long Y, Wang F, Ding JH, Liu SY et al (2005). Systematic administration of iptakalim, an ATP-sensitive potassium channel opener, prevents rotenone-induced motor and neurochemical alterations in rats. J Neurosci Res $\mathbf{8 0}$ 442-449.

Yang Y, Liu X, Long Y, Wang F, Ding JH, Liu SY et al (2006). Activation of mitochondrial ATP-sensitive potassium channels improves rotenone-related motor and neurochemical alterations in rats. Int J Neuropsychopharmacol 9: 51-61. 